\title{
Memory gaps, lost words and crucial mistakes - men's experiences of cognitive difficulties in fibromyalgia
}

\section{Introduction}

Perceived cognitive dysfunction is a common complaint among patients with fibromyalgia. In addition to widespread chronic pain and fatigue, cognitive deficits are included in the current diagnostic criteria of fibromyalgia by the American College of Rheumatology ${ }^{1}$. In fibromyalgia, the patients often describe cognitive difficulties as 'fibro-fog', which may include a perceived decline in mental clarity, increased forgetfulness, and difficulties in solving problems ${ }^{2,3}$. However, in this article we use the term 'dyscognition' as suggested by Glass ${ }^{4}$ and Williams 5 .

The research on cognitive symptoms in fibromyalgia has assessed memory with performance-based tests and questionnaires. According to Glass ${ }^{4}$, patients with fibromyalgia have difficulties in at least three different types of memory tasks: working memory, episodic long-term memory and semantic memory. Working memory is short-time storage for a small amount of information while performing other mental processes, whereas episodic long-term memory is used to remember particular episodes and experiences. Semantic memory involves facts and knowledge about the world, e.g. the meanings and relations of words. Glass ${ }^{6}$ emphasized that a small deficit in working memory may have a large impact on performance in complex tasks. When working memory deficits are combined with deficits in accessing stored knowledge (i.e., semantic memory), it may be difficult for the patient to think quickly or respond with the right word in a given situation. Cognitive deficits related to processing speed and verbal fluency, which have been found in fibromyalgia ${ }^{7}$, seem to leave these patients 'a beat behind', which may be particularly disturbing in work-related discussions and daily decisionmaking situations ${ }^{8,9}$.

In comparison to patients with other rheumatic conditions or healthy age-matched controls, patients with fibromyalgia have significantly poorer results in performance-based memory tests and they report higher levels of anxiety and worry about their memory ${ }^{2,5,10,11}$. Katz et al. ${ }^{2}$ compared self- 
reported memory deficits in 57 patients with fibromyalgia with those of 57 patients with other rheumatic conditions. About $70 \%$ of fibromyalgia patients reported memory problems, in comparison to $24 \%$ of patients with other conditions. The researchers pointed out that one third of patients with fibromyalgia reported a combination of memory problems, mental confusion and speech difficulties, in comparison to only one patient in the group with other rheumatic conditions.

Furthermore, research shows that individuals with fibromyalgia tend to be more susceptible to distraction in comparison to participants without fibromyalgia ${ }^{4,12}$. This might explain why patients often report poor memory performance in real-life situations, where attention needs to be constantly divided between different tasks. Moreover, Verdejo- Garcia et al. ${ }^{12}$ pointed out that patients with fibromyalgia seemed to be less able to develop useful decision-making strategies than the healthy participants.

In qualitative studies, the impact of dyscognition on patients' daily life is rarely discussed as most studies have focused on the impact of pain and fatigue ${ }^{9}$. In a metasynthesis by Sim and Madden ${ }^{3}$, dyscognition was briefly discussed under the topic 'psychological problems', and was described in terms of limitations in problem-solving and abstract thinking. Sallinen ${ }^{13}$ interviewed women with fibromyalgia and noticed that memory difficulties and concetration problems were often seen as a result of poor sleep or as a side-effect of medication and that they were rarely perceived as a cause of work-related disability. In a recent article Sallinen and Mengshoel ${ }^{14}$ pointed out that men with fibromyalgia saw that daytime tiredness and cognitive challenges interfered substantially with the perceived work ability. Similarly, Paulson et al. ${ }^{15}$ noticed that men sometimes felt like outsiders at work place, partially due to cognitive difficulties that resulted in mistakes in performing their duties. Thus, it seems that men and women experience dyscognition and its impact on daily life differently. According to a recent epidemiological study where the new diagnostic criteria ACR2010 were used, the amount of men with fibromyalgia was higher than in earlier studies, and the gender difference in prevalence was not statistically significant ${ }^{16}$, although in clinical populations majority of the patients are still women in their mid-life ${ }^{17}$. The upcoming high number of men with fibromyalgia requests a 
specific focus on men in research to look for gender similarities and particularities because men and women might perceive, express and interpret their illness differently.

\section{Purpose of the study}

The purpose of this study was to explore the impact of perceived cognitive problems on daily life from the vantage point of men with fibromyalgia. The research questions were: 1) what kind of cognitive difficulties the men with fibromyalgia have experienced and 2) how do they express and interpret the impact of dyscognition on their daily life.

\section{Ethics}

The study was conducted in accordance with the Helsinki Declaration. This study is a part of a wider research project conducted in Finland and Norway and therefore research plan and protocol were approved by the local ethical committees in both countries (Board of Ethics of Higher Education Institutions in Satakunta, Finland, 3.12.2016, and Regional Ethical Committee of Research, Sør-Øst D 2016/2242, Norway). Written consent was asked and obtained from all participants, and before the data collection they were reminded about their right to withdraw from the study at any point.

\section{Method}

In narrative approach, storytelling is understood as a fundamental way for humans to give meaning to their own experiences ${ }^{18}$. A chronic illness like fibromyalgia disrupts the coherence and continuation of the life story and can be understood as a 'biographical disruption' that threatens not only the body and mind, but also the sense of self as it was before the illness ${ }^{19}$. According to Mattingly and Lawlor ${ }^{20}$, life stories provide an insider perspective on the invisible; for example, what it is like to be the person who experiences the suffering. Hence, illness narratives provide a way for the patients to explain, contextualize and reflect upon their situation ${ }^{19}$.

\section{Participants}


In qualitative research non-probability sampling methods are usually used to choose the participants for the study. According to Carter et al ${ }^{21}$ purposive sampling refers to selecting information-rich cases for study in-depth. Purposive sampling involves selecting the participants not for representativeness, but for diversity of views. The informants should have experience and insights on the given topic, and they should be able and willing to share their views. Following the ideas of Malterud et al ${ }^{22}$ we anticipated that sufficient 'information power' would be achieved through sample specifity, in-depth interviews and rigorous analysis rather than through a large amount of participants. Therefore, we aimed at reaching 6-12 men with different experiences of living with fibromyalgia for interviews.

In Finland the invitation to participate to the study was sent through five local rheumatism associations and through the Finnish fibromyalgia association's Facebook on-line support group. In Norway the invitation was sent through a local rheumatism association and through a rehabilitation center for patients with musculoskeletal problems. The patients from the rehabilitation center were anticipated to be newly diagnosed whereas patients from support groups would presumably have a longer history with fibromyalgia.

The invitation described the study plan and purpose, and men with fibromyalgia were asked to contact the researchers if they were interested in participating. Altogether ten men with fibromyalgia responded to the invitation within the given time. However, one man withdrew from the study before data collection and one was eventually not reached by phone or e-mail.

\section{Data collection and analysis}

We contacted each participant first by phone to agree on a convenient time and place for the interview. Most interviews were conducted at the participants' home or place of work, one in a café and one at the university. In the beginning of each interview, the information sheet about the study was revisited and the consent form was filled in and signed. Moreover, permission to record the 
interviews was asked and the participants were reassured that the audio recorder could be switched off if necessary.

We used a 3-phase interview method inspired by Rosenthal ${ }^{23}$. First, the interviewer invited the participant to tell his life story in his own words. In this phase, the interviewer did not interrupt but showed active and empathetic listening. In the second phase, the interviewer asked questions that had emerged during the storytelling to enrich the life story (for example, 'could you tell me more about that situation'?) or to fill in gaps in the story (for example, 'where did you work before this'?) Finally, the interviewer closed the session with a discussion about the contribution and the interview session as a whole. The length of the interviews ranged from one to three hours. The recorded data were transcribed verbatim for the analysis.

According to Polkinghorne ${ }^{24}$, analysis in narrative research may include paradigmatic analysis of the narratives and narrative analysis. Paradigmatic analysis seeks to locate common themes or conceptual manifestations among the told stories. In narrative analysis, the task for the researcher is to develop a plot that unites the individual stories and displays a linkage among the elements as parts of an unfolding temporal development, for which we use the term 'model narrative'. In the present study, temporal development is explored through how the participants reflect on their past and present experiences and discuss their anticipated future.

Immediately after each interview the main content and preliminary impressions and reflections were shared and discussed between the researchers to ensure relevance of the data and transparency of the process. The recorded data was listened to several times alongside the transcripts to grasp the 'whole' of the data and to check the accuracy of the transcripts. Some notes about the atmosphere or intensity of the storytelling were made at this point. Because we were particularly interested in cognitive functioning, all episodes about difficulties in cognition or their consequences in daily life were collected in a separate file. In the analysis process we were interested both in the content and the ways how men explicated their experiences; what kind of wordings, metaphors and examples were used to emphasize the contents? Furthermore, we looked how the episodes were linked to the storyline of the 
given narrative to analyze the impact of cognitive deficits on work ability, social life and daily functioning as well as on the anticipated future.

\section{Results}

A total of eight men took part in the interviews. They were on average 47 years old (range 24-61) and had been diagnosed with fibromyalgia on average 9 years ago (range 1 month-20 years). They had all had symptoms for several years prior to the diagnosis. Six participants were married/cohabiting and had children and two were single. Four of the men had bachelor level education, three had vocational education, and one had only basic education. Three participants worked full time, while another two were on reduced working hours. Three participants were on permanent or temporary disability pension.

The cognitive challenges the participants had experienced were discussed in all the interviews. In some cases, dyscognition was the most prominent symptom of fibromyalgia in addition to pain, whereas in some others it was referred to as 'minor inconvenience'. The scope and intensity of dyscognition, as well as how much it disturbed one's life and work varied form one patient to another. Moreover, the interviewees expressed that the intensity of cognitive problems could fluctuate unexpectedly from one day to the next.

The results of the narrative analysis are presented in the form of three model narratives that comprise the content and the storylines of the original accounts of the interviewees. We named them 'Story of focusing on one thing at a time', Story of having memory gaps' and 'Story of losing cognitive work ability'.

In the following examples, we notate pausing in speech with dots (...) and emphasis of words with underlining. To help the reader to understand the quotations we also provide some additional information in square brackets [e.g., expressed feelings or context]. Omitted text is notated with three dots in square brackets [...]. In addition, we gave all participants a pseudonym (from A to H), and some identifying details have been changed to protect the privacy and integrity of the participants. 


\section{Story of focusing on one thing at a time}

In the 'Story of focusing on one thing at a time' cognitive difficulties were mild and explicated only in the context of intensive pain periods. The men used focusing on the task-at-hand as a selfmanagement strategy to exclude the intrusive pains. This meant that one tried to distract thoughts away from the pain by consciously concentrating on the activity one was doing at the given moment. However, when the pains and aches were more intense this seemed to be more difficult.

...it is often said that men only can do one thing at a time... that is exactly what I try to do on purpose... if I am reading a book, I focus on reading and try to exclude everything else...but sometimes when the pains are intense and you are more tired...[...] it is difficult... and for example, when my daughter wants me to look at her drawing and I am just like...' whatever, couldn't care less'... I just cannot concentrate on that then... (Alex, age 24)

The two men (Alex and Ben) who presented this kind of a story did not acknowledge cognitive problems other than occasional absent-mindedness that everyone experiences every now and then. The following extract illuminates this aspect:

I have always had quite good concentration skills... but on the other hand, I had to ask you [earlier during the interview] 'what was the question again? [laughs] ... but anyway, I don't think I have any problems there...(Ben, 43,)

The cognitive difficulties did not disturb the daily life, although often more effort was needed to be able to concentrate on work tasks, for example. Moreover, the men did not anticipate having cognitive problems in the future if the pains were tolerable. They saw controlling the pain and sleep as a key to a better life quality in general. The men anticipated to have a 'normal life' despite fibromyalgia: to be able to work, to return to their earlier hobbies and to have enough energy for the family.

\section{Story of having memory gaps}


'Story of having memory gaps' describes mild to moderate, yet frequent cognitive difficulties and how they affect one's life, work and future perspectives. Three men (Christer, Dan and Ed) with a long history of fibromyalgia gave an account with a parallel storyline. In the life stories of these three men, the cognitive problems were expressed as recurrent difficulties in finding words, having occasional memory problems and difficulties in concentration. For example, reading books or newspapers took time because one had to go back in text since one could not remember what was said in the previous chapter. Experiences of not being as 'sharp' as before were frequently expressed in the accounts. The cognitive problems were often linked to poor sleep, daytime fatigue and intense pains. The following extract sheds light on the perceived memory gaps, or as Christer says, 'blackouts'.

If you have had a bad day or done something strenuous, the pain hits you hard... and when it continues for a couple of days you may have 'black-outs'... you are explaining something and suddenly you don't have a clue what you were talking about ... Even if the others try to help you out you cannot reach the thought you had... it is annoying... (Christer, 45)

However, sometimes the memory gaps came unexpectedly, without any obvious connection to other symptoms, which was problematic as one did not have any tools to improve the situation.

I am not necessarily more in pain or more tired then... it can happen whenever... [...] as if there was no blood flow in the brain ... it's not always linked to pains or other symptoms... if it was, I would know that I have to take it easier now and try to get better somehow.... (Ed, 51)

The men described dyscognition as annoying, embarrassing and frightening. They described vividly how embarrassing it was in work contexts, when, for example, they suddenly forgot the name of a colleague or customer in the middle of discussion, or what one was about to do or say, and sometimes it took a long time to get back on track. Furthermore, the cognitive problems also affected family life and sometimes caused friction in the relationship with the spouse. Dan's example illustrates this aspect: 
Oh, my wife should be here now to tell you! [Laughs] ... she is always complaining about my poor memory... she gives me a list for shopping and I may end up coming home with only half of the items I was supposed to buy! Then I have to go back! (Dan, 61)

The men referred to a fear of having more serious memory problems in the future. Christer explicated this clearly:

It's annoying when it comes all of a sudden... it is as if you had early dementia, Alzheimer's
or something ... it is very disturbing... and frightening, too.

\section{Story of losing cognitive work ability}

In the life stories of three men, Freddie, George and Harry, the focus was on experienced or anticipated loss of work ability due to severe cognitive difficulties. In their life stories, the scope of dyscognition varied from severe concentration and memory problems to difficulties in problemsolving and making crucial mistakes at work.

In the 'Story of losing cognitive work ability', tasks that were previously easy routines that could almost be performed with eyes closed had become increasingly difficult. It took much longer than before to complete even simple tasks, and sometimes one had to ask for help from colleagues.

Solving complex problems was sometimes experienced as impossible.

You see, I have trained all the other employees here and taught them everything about these systems and solutions and now.... eehh.... sometimes I just must go and ask someone else to show me how this and that is done... and they are like 'Are you joking?'... you see, these are things that I used to know well... (George, 55)

Problems in concentration and memory often caused awkward and embarrassing situations in meetings with colleagues and clients. The men described vividly how strenuous it was to follow discussions, let alone remember afterwards what had been planned, agreed and decided. The men also talked about dyscognition in terms of difficulties in planning the workday. Sometimes they took 'the 
easy way out' because they were not able to perform complex tasks, could not decide what to do during the day, or were not able to prioritize tasks. This was often associated with an increase in other symptoms or with stress and pressure at work. The following extract illustrates this issue:

...well, I had to drag myself to the office after a poorly slept night... feeling very tired...I was not able to plan my work... I did what I thought was important... or what was easiest to do... [...] ... We had quite a lot of pressure those days... you had to have results... and the tighter the spot was, the worse was my performance... (Harry, 43)

One of the men, Freddie, was on disability pension due to severe cognitive difficulties. His work ability had been examined from different perspectives during a work try-out. In a neuropsychological test, he failed to continue the tasks under pressure. He reflected upon the situation:

....one clear feature there was that my concentration was like a rubber band...it could be stretched for a while but then it just snapped... and when there was more time pressure and so on... I could not continue at all, but kind of 'froze' completely ... and in any job you should be able to perform under pressure, you know...(Freddie, 51)

Together with problems in concentration and memory, perceived distress and pressure could result in mistakes at work. The men pointed out that if one was a key-person in the enterprise, there was no room for mistakes, and big mistakes could eventually lead to losing one's job.

For example, I had negotiated a contract with a customer but had forgotten to add some regular costs... that are always added there on top of everything else... of course I noticed the mistake the minute the order came... but it was too late then because the contract was already approved and signed... we got no profit from that order ... [...] ...I don't know how long my employer will turn a blind eye to mistakes of this kind...(George, 55)

The fear of losing one's work ability was reflected upon from different vantage points in the interviews. It was worrying and frightening for the men to notice that they could not perform on the same level as earlier due to the dyscognition. It was perceived as embarrassing when the colleagues noticed the cognitive difficulties and expressed their concern about one's work ability. 
[For me] it was easy to put the pains and aches aside... but when your colleague says that now there is something wrong with you, are you losing your marbles or what?... you cannot remember what we just discussed two minutes ago... it was a wake-up call... (Harry, 43)

The men expressed that the role they had in the work community had changed dramatically as the work ability deteriorated. They described vividly the transition from 'a key-person to an auxiliary worker' in the enterprise, and how difficult it was for them to accept the perceived decrease in work ability. At some point in the life stories of these three men, the future at work had started to appear uncertain due to the cognitive difficulties. They were not only worried about losing their work ability, but also about losing their job and part of the 'self' with it. George pondered his current situation as follows:

I used to be the one that knew these things best... and now I am regarded as a resource person [auxiliary worker] ...I don't know if they really need me anymore ...[...] This is the job I have done all my life and I don't know about anything else...I don't know what is to come... I can manage financially and so on ... but work has been such a big part of my life so far...all this scares the hell out of me! (George, 55)

In contrast to this, Harry had recovered well and was now able to work on the same level as before the symptom onset. He explicated that this was a result of suitable medication and substantial increase in physical activity, which had helped him to manage his pains and sleeping problems better than earlier.

Of course, it doesn't make any sense to work 17 hours a day like I did a couple of weeks ago...but it shows that I am able to do that now....earlier, my input was maybe $20 \%$ [of the expected]

\section{Discussion}


The present study aimed at shedding light on experiences of cognitive problems among men with fibromyalgia. The results show that the impact of dyscognition on the daily life of men with fibromyalgia was in many cases substantial. The experienced cognitive deficits affected not only their work performance, but also domestic chores, social relations and self-perception. However, the scope of dyscognition varied individually from occasional concentration problems to severe and complex difficulties in memory, concentration and problem solving.

According to Williams et al. ${ }^{5}$, the effort required to perform cognitive tasks may influence the individual's perception of cognitive ability even when the performance is not affected per sé. In the present study, the 'story of focusing on one thing at a time' describes such a situation. Although the cognitive deficits were not apparent or recognized, the men felt that more effort was needed to be able to concentrate when pains were more intense and intrusive.

In the second model narrative, 'Story of having memory gaps', frequent cognitive difficulties overshadowed the daily lives of the participants. The men in our study seemed to be able to make a clear distinction between cognitive problems that were related to pain or insomnia and those that had no obvious association with other symptoms. The memory gaps that could not be explained by pains or sleepless nights caused additional worry and anxiety about the underlying mechanisms of the cognitive deficits. As suggested in earlier studies ${ }^{4,6}$, these experiences might reflect a manifestation of a combination of deficits in working memory and semantic memory that particularly affect verbal fluency, and they are not an indication of early dementia as such ${ }^{25}$. However, the fear and anxiety of a severe cognitive illness that were vividly expressed in our data are justified and worth further research. It is noteworthy that although six of the eight interviewees experienced cognitive difficulties frequently, only one of them had been subjected to neuropsychological examinations. This implies that these kind of moderate-to-severe cognitive problems experienced by patients with fibromyalgia are not recognized, or are underrated in health care.

Bearing in mind that most of our interviewees worked or had worked in jobs where high cognitive performance level was expected, it is not surprising that difficulties in problem-solving, verbal fluency or memory had a substantial impact on their work performance. Especially comments coming 
from work colleagues seemed to be of importance in acknowledging that cognitive functioning was not on the same level as before. This seemed to collide with the earlier identity of 'a man with knowledge and skills' and lead to substantial changes in one's social roles at work, in the family and in the wider community. In health sociology, the concept 'biographical disruption' is used to describe a situation where the anticipated course of life is disrupted in a way that forces one to re-evaluate one's life, identity and future with the illness and despite $i^{18}$. In our data, the cognitive problems were at first interpreted as common forgetfulness or absent-mindedness that everyone experiences occasionally. However, when these problems became more frequent and more severe, the interpretation of 'normality' of the problem came to a dead end and re-evaluation of the whole life situation and the 'self' was needed. Our findings about the struggle to retain the role in working life accord with and expand the knowledge about cognitive problems in fibromyalgia patients' daily lives reported in earlier studies $2,9,13,14,15$.

The question whether men with fibromyalgia have different experiences of dyscognition in comparison to women is interesting. It is possible that the cognitive problems as such are similar but the way of explicating and reflecting on them is different. The concept 'fibro-fog' that is widely used to describe the experiences of dyscognition is rooted in patient parlance - presumably used by female patients. It gives an impression of obscure and indefinite mental confusion and vague memory problems that may not reach the attention of health care professionals. However, in our study the descriptions of cognitive deficits were all but obscure: precise, detailed, to-the-point descriptions of a variety of different problems supported with vivid examples of real-life situations. Based on our results we suggest that instead of settling for the somewhat mystifying expressions of 'fog', the health care professionals should address difficulties in problem solving, speech fluency, memory and concentration in more detail to be able to find specific interventions that would be beneficial and relevant for the patient

We had an all-male sample, whereas in the majority of earlier qualitative studies, the participants were female or the number of male participants is low ${ }^{3}$. Moreover, to our knowledge, cognitive functioning has not been in the focus of earlier qualitative studies. Rather than that, earlier studies have explored 
the impact of fibromyalgia on patients' lives more broadly $9,13,14,15$. Thus, our findings bring new insights to experiences of dyscognition among men with fibromyalgia, and more widely, to experiences about living with fibromyalgia.

In this study, narrativity was used both as a philosophical approach and as a method of collecting data. Although the research project was perceived as important and interesting and raised a lively discussion for example among one of the support group in Facebook, we failed to reach large amounts of men with fibromyalgia. Thus, the sample may be biased, and it is possible that some other experiences than those discussed here would emerge in a bigger sample. Malterud et al ${ }^{22}$ pointed out that sample adequacy, data quality, and variability of relevant events are often more important than the number of participants. In research interviews, the impact of the interviewer is inescapable; the richness of data depends on the quality of interaction and trust between the participant and the interviewer ${ }^{22}$. In this study both interviewers were female but had a long experience about working with both male and female patients as physiotherapy professionals and as researchers. The interviewers were obviously perceived as trustworthy and easy to talk to since the men disclosed also sensitive and difficult events in their accounts. The practical examples, precise wordings and lively metaphors the participants used to expound their cognitive difficulties gave us rich data which allowed versatile and profound analysis Thus, reflecting on the work of Malterud et al. ${ }^{22}$ we believe that the information power of the data was sufficient for the purposes of this study.

In conclusion, the men in this study had experienced a wide scope of cognitive problems, in which the severity varied from minor to severe, and the frequency from occasional to constant. The dyscognition experienced by men with fibromyalgia seemed to have a substantial negative impact on their daily lives, work and social relations. For some participants, a future with dyscognition appeared as uncertain and frightening, and in some cases maintaining the paid work was perceived as challenging. In the future, more research on effective rehabilitation options is needed to develop interventions for primary care where the patients with dyscognition associated with chronic pain are usually examined and treated. Furthermore, we suggest that the assessment and interventions should address not only 
memory, but also individual's ability to solve complex problems and to work under pressure as well as the level of verbal fluency, which all are relevant issues in terms of work ability in contemporary society.

\section{Acknowledgements}

The research leading to these results has received funding from the European Union Seventh Framework Programme (FP7-PEOPLE-2013-COFUND) under grant agreement $n^{\circ} 609020$ - Scientia Fellows.

\section{Conflict of interest}

The authors declare that there are no conflicts of interest.

\section{References}

1. Wolfe F, Clauw DJ, Fitzcharles MA, et al. The American College of Rheumatology preliminary diagnostic criteria for fibromyalgia and measurement of symptom severity. Arthritis Care Res (Hoboken). 2010; 62: 600-10.

2. Katz RS, Heard AR, Mills M and Leavitt F. The prevalence and clinical impact of reported cognitive difficulties (fibrofog) in patients with rheumatic disease with and without fibromyalgia. JcrJ Clin Rheumatol. 2004; 10: 53-8.

3. $\quad$ Sim $\mathbf{J}$ and Madden S. Illness experience in fibromyalgia syndrome: a metasynthesis of qualitative studies. Soc Sci Med. 2008; 67: 57-67.

4. Glass JM. Cognitive Dysfunction in Fibromyalgia Syndrome. Journal of Musculoskeletal Pain. 2010; 18: 367-72.

5. Williams DA, Clauw DJ and Glass JM. Perceived Cognitive Dysfunction in Fibromyalgia Syndrome. Journal of Musculoskeletal Pain. 2011; 19: 66-75.

6. Glass JM. Review of Cognitive Dysfunction in Fibromyalgia: A Convergence on Working Memory and Attentional Control Impairments. Rheum Dis Clin N Am. 2009; 35: 299-+.

7. Leavitt F and Katz RS. Cognitive Dysfunction in Fibromyalgia: Slow Access to the Mental Lexicon. Psychol Rep. 2014; 115: 828-39.

8. Kravitz HM and Katz RS. Fibrofog and fibromyalgia: a narrative review and implications for clinical practice. Rheumatol Int. 2015; 35: 1115-25.

9. Arnold LM, Crofford LJ, Mease PJ, et al. Patient perspectives on the impact of fibromyalgia. Patient Education and Counseling. 2008; 73: 114-20.

10. Glass JM, Park DC, Minear M and Crofford LJ. Memory beliefs and function in fibromyalgia patients. J Psychosom Res. 2005; 58: 263-9.

11. Park DC, Glass JM, Minear M and Crofford LJ. Cognitive function in fibromyalgia patients. Arthritis and Rheumatism. 2001; 44: 2125-33.

12. Verdejo-Garcia A, Lopez-Torrecillas F, Calandre EP, Delgado-Rodriguez A and Bechara A. Executive Function and Decision-Making in Women with Fibromyalgia. Arch Clin Neuropsych. 2009; 24: 113-22. 
13 Sallinen M. 2012. Women's narratives on fibromyalgia, functioning and life events Jyväskylä: University of Jyväskylä. Studies in Sport, Physical Education and Health 180.

14. Sallinen M \& Mengshoel AM. "I just want my life back"- Men's narratives about living with fibromyalgia. Disabil Rehabil. 2017 Oct 26:1-8. doi: 10.1080/09638288.2017.1395085

15. Paulson M, Danielson E and Soderberg S. Struggling for a tolerable existence: the meaning of men's lived experiences of living with pain of fibromyalgia type. Qual Health Res. 2002; 12: 238-49.

16. Wolfe F, Brahler E, Hinz A and Hauser W. Fibromyalgia prevalence, somatic symptom reporting, and the dimensionality of polysymptomatic distress: results from a survey of the general population. Arthritis Care Res (Hoboken). 2013; 65: 777-85.

17. Hauser W, Kuhn-Becker H, von Wilmoswky H, Settan M, Brahler E and Petzke F.

Demographic and clinical features of patients with fibromyalgia syndrome of different settings: a gender comparison. Gend Med. 2011; 8: 116-25.

18. Garro L, Mattingly, C. Narrative as Construct and Construction. In: Mattingly CG, LC, (ed.). Narrative and the cultural construction of illness and healing Berkeley California Press, 2000, p. 149.

19. Hydén L-C. Illness and narrative. Sociology of health \& illness. 1997; 19: 48-69.

20. Mattingly $C$ and Lawlor M. Learning from Stories: Narrative Interviewing in Cross-cultural Research. Scand J Occup Ther. 2000; 7: 4-14.

21. Carter R, Lubinsky J and Domholdt E. Rehabilitation research. Principles and applications. Elsevier, 2011.

22. Malterud K, Siersma V and Guassora A. Sample Size in Qualitative Interview Studies: Guided by Information Power. Qualitative Health Research 2015: 1-8. DOI: $10.1177 / 1049732315617444$

23 Rosenthal G. The healing effects of storytelling: on the conditions of curativestorytelling in the context of research and counselling. Qualitative Inquiry,. 2003; 9: 915-33.

24. Polkinghorne DE. Narrative configuration in qualitative analysis. International Journal of Qualitative Studies in Education. 1995; 8: 5-23.

25. Leavitt F and Katz RS. Cross-sectional Neurocognitive Data Do Not Support a Transition From Fibrofog to Alzheimer Disease in Fibromyalgia Patients. Jcr-J Clin Rheumatol. 2015; 21: 81-5. 